\title{
La catégorie de l'aspect en français
}

\author{
Tatiana Milliaressi \\ UMR 8163 STL, CNRS \& Université Charles de Gaulle Lille III \\ tatiana.milliaressi@univ-lille3.fr
}

\section{Introduction}

L'aspect est une catégorie universelle exprimée de façon différente dans chaque langue. L'expression prototypique de la catégorie grammaticale de l'aspect est traditionnellement attribuée aux langues slaves où l'aspect (imperfectif/perfectif) du verbe est marqué morphologiquement (voir plus bas). Dans cet article, en faisant référence aux propriétés sémantiques de l'aspect slave (sur l'exemple du russe), j'essayerai de répondre à la question suivante : la catégorie de l'aspect se présente-t-elle en français en tant que catégorie grammaticale?

La réponse à cette question est liée à la compréhension et à la définition du sens grammatical de la catégorie de l'aspect et des procédés formels de son expression.

L'aspect est défini comme la façon de représenter le déroulement du procès dans le temps :

[...] les aspects sont les manières diverses de concevoir l'écoulement du procès même. (Holt $1943: 6$ )

Aspects are different ways of viewing the internal temporal constituency of a situation. (Comrie 1976: 3)

L'aspect est une catégorie grammaticale qui exprime la représentation que se fait le sujet parlant du procès exprimé par le verbe (ou par le nom d'action), c'est-à-dire la représentation de sa durée, de son déroulement ou de son achèvement (aspects inchoatif, progressif, résultatif, etc.)... (Dubois \& al. 1994 : 53)

[...] ASPECT (repérage non déictique de la DURÉE et des LIMITES du procès) [...] (Laurendeau 1995)

Ces citations mettent en évidence la double nature sémantique de l'aspect : d'une part, l'aspect peut être compris comme une opposition de la durée du procès (1a) par rapport à la délimitation de cette durée (1b) ; d'autre part, comme le déroulement du procès (2a) par rapport à son aboutissement, résultat, télos (2b) :

(a) Paul travaillait à cette époque.

(b) Paul a travaillé aujourd'hui.

(2) (a) Paul mangeait des fraises, Marie lui a proposé de la Chantilly.

(b) Paul a mangé une fraise.

Cette dualité aspectuelle mérite d'être éclaircie: est-ce que la télicité est une propriété inhérente à l'expression de l'opposition aspectuelle (imperfectif/perfectif, inaccompli/accompli, non délimité/délimité) ? J'essayerai de répondre à cette question dans le $\S 2$.

La mise en forme grammaticale du sens aspectuel n'est pas toujours clairement définie non plus, ce qui crée une confusion entre la valeur grammaticale de l'aspect (marquée de façon régulière par un affixe, une flexion ou une forme analityque) et l'expression lexicale de l'aspectualité (Aktionsart) (exprimée par le radical du mot avec ou sans préfixe à sens lexical ${ }^{1}$ ).

La régularité de l'expression morphologique de l'aspect pour tout le système verbal des langues slaves est à l'origine du point de vue répandu selon lequel la valeur aspectuelle grammaticale doit être transmise exclusivement par la base verbale (voir, par exemple, Peškovskij, 1956 : 105 ; Jacobs \& Rosenbaum, 
1968 ; Lyons, 1970 : 242), comme c'est le cas en russe. Dans cette optique, l'aspect n'est pas une catégorie grammaticale dans les langues à tendance analytique comme le français.

En effet, en russe, par opposition au français, l'aspect est une caractéristique morphologique inhérente au verbe où chaque forme verbale russe est associée à l'un des aspects (perfectif ou imperfectif). À l'exception d'un nombre restreint de verbes biaspectifs (verbes qui selon le contexte peuvent avoir un sens perfectif ou imperfectif) et des verbes sémantiquement sans terme naturel (imperfectiva tantum) ou sémantiquement résultatifs (perfectiva tantum), le verbe russe se présente sous la forme d'un couple aspectuel :

- « écrire » : pisat' (imperfectif) / $\underline{\text { napisat' }}$ (perfectif par préfixation) ${ }^{2}$;

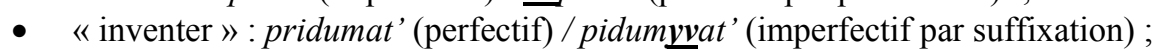

- « décider » : reš $\underline{\underline{i}}{ }^{\prime}$ ' (perfectif) / reš $\underline{\underline{a} t} t^{\prime}$ (imperfectif par changement de voyelle thématique);

- «prendre » : $\underline{\text { brat }}$ ' (imperfectif) / $\underline{\boldsymbol{v} z j a t}$ ' (perfectif par supplétion des bases) ${ }^{3}$.

Cette opposition aspectuelle en russe est indépendante de son expression temporelle. En effet, l'infinitif est doté déjà du sens aspectuel (imperfectif ou perfectif). L'opposition aspectuelle russe est systématique pour les temps du passé (passé imperfectif / passé perfectif) et du futur (futur imperfectif / futur perfectif). Le présent est marqué par l'imperfectif et ne présente donc pas d'opposition aspectuelle.

En français, la valeur aspectuelle n'a pas d'expression autonome : les marques temporelles et aspectives sont présentées de façon syncrétique. En revanche, l'opposition des temps grammaticaux dans le cadre du même tiroir (passé) qui expriment prototypiquement la durée (par exemple, imparfait) par rapport aux temps transmettant une délimitation de cette durée (par exemple, passé composé, passé simple) peut être analysée comme une opposition aspectuelle (Guillaume, 1951; Klum, 1961 ; Imbs, 1965 ; Cohen, 1989 ; Vetters, 1996 ; Gausselin, 1996, 2005, Čertkova, 2004).

À la suite de Maslov (1978: 307) et Lerat (1981: 50), je pense que la nature de l'expression formelle de l'aspect verbal (par sa base, sa flexion ou à l'aide des formes analytiques) n'est pas prioritaire pour être considérée comme une catégorie grammaticale. En revanche, il est important que la totalité ou la plus grande partie du lexique verbal présente des oppositions systématiques de valeur aspectuelle grâce aux paradigmes des formes grammaticales du même verbe: en russe, l'opposition des paradigmes de conjugaison des formes perfectives par rapport aux formes imperfectives du même verbe au passé et au futur (passé perfectif / passé imperfectif, futur perfectif / futur imperfectif); en français, l'opposition des temps grammaticaux du même tiroir qui expriment le procès dans sa durée par rapport à ceux qui marquent le procès à durée délimitée (passé composé, passé simple / imparfait ; futur antérieur / futur simple). J'utiliserai ici les termes de D. Cohen, aspect non délimité et aspect délimité, qu'il propose comme termes génériques pour diverses langues (Cohen, 1989: 71), afin de distinguer l'expression de la durée du procès et la délimitation de cette durée.

En français, l'opposition aspectuelle est déficiente pour les temps du futur, celle-ci n'étant valable de façon systématique que pour les temps passés. Je m'intéresserai donc tout particulièrement dans cet article à l'expression de la valeur aspectuelle au passé.

\section{La télicité aspectuelle en russe}

La télicité est une propriété des procès pourvus d'une limite intrinsèque appelée télos; lorsque le télos est atteint, le procès a abouti et ne peut plus continuer, à moins de recommencer : arriver, se réveiller, tomber, construire (une maison). Ainsi, les procès pourvus d'un télos sont téliques. Par conséquent, les procès dépourvus d'un télos sont atéliques : se promener, marcher, dormir, réfléchir.

Étant une des propriétés essentielles de l'aspect slave, la télicité est souvent considérée comme une propriété universelle de l'aspect perfectif (Smith, 1986 ; Comrie, 1989 ; Bondarko, 1987 ; Šeljakin, 2001 ; Breu, 2004 : 252). En effet, en dehors de l'opposition duratif/délimitatif, l'aspect russe marque en plus l'opposition télique/atélique. Dans ce qui suit, j'essayerai de démontrer tout d'abord que la télicité est une 
des propriétés essentielles mais non exclusive de l'aspect russe, et ensuite d'analyser le rôle de la télicité dans l'expression aspectuelle en français.

Les procès duratifs téliques en russe présentent une opposition de la forme imperfective et de la forme perfective du même verbe ce qui reflète la conceptualisation du monde propre à la langue russe. Ainsi, le procès « lire » est conceptualisé comme une durée (processus) avec une fin (télos). La durée sans télos est exprimée par l'imperfectif; elle peut être non délimitée (3a) ou délimitée (4a). La durée avec télos est marquée par le perfectif (5a). ${ }^{4}$

(3) (a) Kogda Marija vošla v komnatu, Viktor čital (imperfectif passé) knigu.

(b) Quand Marie est entrée dans la pièce, Victor lisait (aspect non délimité) un livre.

(a) Čto ty delal včera večerom ? - Ja čital (imperfectif passé) knigu.

(b) Comment as-tu passé ta soirée hier? - J'ai lu (aspect délimité) un livre. ('le livre n'est pas lu')

(a) Včera večerom ja pročital (perfectif passé) ètu knigu.

(b) Hier soir, j'ai lu (aspect délimité) ce livre. ('le livre est lu')

Les exemples montrent que l'imperfectif passé russe peut marquer la durée non délimitée (3a), ainsi que la durée délimitée $(4 a)$. Le procès télique de l'exemple $(5 a)$ représente une durée délimitée où la borne finale coïncide avec le télos du procès (voir Milliaressi, $2006: 555$ sqq.).

On associe souvent à tort le procès télique à l'aspect perfectif et le procès atélique à l'aspect imperfectif (voir, par exemple, Cohen, 1989: 13). En réalité, la relation est plus complexe. Il me semble indispensable de superposer les deux approches: la délimitative (appliquée aux langues romanes et germaniques) et la télique (appliquée aux langues slaves). En effet, le télos du procès duratif coïncide avec sa fin temporelle : dans l'exemple (5), 'la durée de lecture est finie puisque le livre est lu'. Mais si le procès est atélique, il s'agit d'une délimitation temporelle où le procès a un début et une fin, la fin de ce procès n'implique pas que le télos soit atteint (4). La différence de la délimitation russe par rapport au français est la capacité de transmettre en cas de besoin la télicité du procès (5a). Comparez pročital (5a) avec la forme verbale française ai $l u(4 \mathrm{~b}, 5 \mathrm{~b})$ qui veut dire : 'il y a eu un intervalle de temps pendant lequel j'ai lu' (où l'aspect délimité est exprimé par le passé composé).

Les exemples (3a, 4a, 5a) amènent à la conclusion qu'en russe, ce n'est pas la délimitation de la durée qui nous oriente vers le choix de l'aspect, mais la télicité du procès. En effet, pour mettre en exergue la télicité du procès, on utilisera le perfectif (5a). Cependant, le procès duratif atélique est exprimé en russe par un couple aspectuel verbal (imperfectif/perfectif) ce qui laisse penser que la télicité n'est pas une propriété inhérente au perfectif. En effet, pour les procès atéliques, l'imperfectif passé marque une durée délimitée ainsi qu'une durée non délimité, tandis que le perfectif marque toujours une durée délimitée. Pourquoi avoir deux aspects (perfectif et imperfectif) pour marquer une durée délimitée ?

Examinons les trois emplois essentiels du perfectif pour les procès atéliques qui expriment :

1) une évaluation subjective de la durée ;

2) une évaluation de l'évolution du procès au moment de l'énonciation ;

3) le début du procès.

Analysons chacun de ces trois emplois.

\section{1 Évaluation subjective de la durée}

Lorsque la durée est délimitée par deux bornes temporelles (le début et la fin), le perfectif et l'imperfectif en russe sont interchangeables dans le même contexte. L'imperfectif marque une constatation objective du fait (6), tandis que le perfectif connote une perception subjective de la durée (suffisante, insuffisante, pénible à supporter, etc.) (7) :

(6) Ja rabotal (imperfectif passé) dva časa.

" J'ai travaillé deux heures (simple constatation). » 
(b) Ja porabotal (perfectif passé) dva časa.

« J'ai travaillé deux heures (période perçue comme passée rapidement). »

(c) Ja otrabotal (perfectif passé) dva časa.

«J'ai travaillé deux heures (période prévue au préalable pour le travail). »

Le perfectif dans ce cas est formé à partir de l'imperfectif avec un préfixe délimitatif (par exemple, po-, pro-, ot-). Les préfixes délimitatifs du perfectif russe ont une nature complexe (Milliaressi, 2010). Le sens du perfectif délimitatif russe peut être présenté de façon conventionnelle comme une superposition des trois niveaux :

(i) délimitation d'une durée (sens grammatical de tous les perfectifs russes),

(ii) évaluation d'une durée (sens grammatical de tous les perfectifs délimitatifs russes),

(iii) sens lexical spécifique en fonction du préfixe concret.

Les perfectifs délimitatifs russe jouent un rôle important pour exprimer l'ordre des procès. Les valeurs du perfectif délimitatif par rapport à l'imperfectif peuvent être présentées de la façon suivante :

Le perfectif délimitatif (procès atélique) marque :

(a) une succession des procès délimités ou bien,

(b) une évaluation subjective de la durée par le locuteur.

Quant à l'imperfectif (procès atélique), il marque :

(a) une coöncidence des procès (délimités ou non délimités) et

(b) une constatation neutre du procès (il ne comporte donc pas de sens connotatif).

J'analyserai le rôle de l'aspect pour l'expression de l'ordre des procès dans le $\S 4$. Dans cette section, je m'intéresserai aux valeurs du perfectif par rapport aux procès atéliques.

\section{2 Évaluation de l'évolution du procès}

Les procès évolutifs (non contrôlables ${ }^{5}$ ) comme « augmenter », « baisser », «s'améliorer » sont exprimés en russe par l'imperfectif et le perfectif : l'imperfectif est formé depuis le perfectif par suffixation («augmenter » uveličit'sja (perfectif) / uveličivat'sja (imperfectif)) ou par changement thématique de base : «baisser » ponizít'sja (perfectif) / poniža $\underline{a}$ 's $a$ (imperfectif)). Le perfectif et l'imperfectif ne sont pas interchangeables dans le même contexte : le perfectif marque une évaluation de l'évolution du procès par le locuteur qu'il fait au moment de l'énonciation (8).

(a) Uroven' žizni povysilsja (perfectif passé).

(b) Le niveau de vie a augmenté.

Ainsi, dans l'exemple (8), on interprète prioritairement le procès évolutif comme continu, c'est-à-dire que le niveau de vie continuera à augmenter: le locuteur a évalué l'évolution du procès au moment de l'énonciation. Cependant, il n'est pas exclu que le processus ait atteint la limite dans son évolution et que le niveau de vie n'augmente plus.

\subsection{Inchoativité et ingressivité}

Les procès inchoatifs sont des procès duratifs qui reçoivent une borne temporelle au début. Ils sont différents des deux types de procès atéliques examinés plus haut : ils n'ont pas de borne finale. Cette délimitation initiale est atélique, elle est exprimée morphologiquement en russe: crier/ kričat'

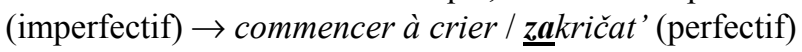


À la différence du perfectif délimitatif (avec un préfixe délimitatif), le perfectif inchoatif (avec un préfixe inchoatif) n'a pas de corrélatif imperfectif en russe : le perfectif inchoatif n'est donc pas interchangeable avec l'imperfectif. Le perfectif inchoatif ne marque pas grammaticalement d'évaluation subjective du commencement de la durée.

Lorsque la borne initiale d'un procès duratif ne constitue pas seulement le début du procès mais représente un changement qualitatif et qu'elle engendre un procès duratif, il s'agit d'un procès ingressif. L'ingressivité par rapport à l'inchoativité peut être définie comme un commencement d'un procès intense perçu et évalué par le locuteur comme brusque, subit. Par exemple : s'éprendre présuppose le procès duratif « être amoureux, aimer », voznenavidet" « commencer subitement à haïr».

Souvent, l'inchoativité est considérée comme atélique, tandis que l'ingressivité est télique. Je pense que le sens connoté (évaluation subjective du commencement du procès) ne peut pas être considéré comme le télos : en effet, le procès perdure au-delà de la borne initiale. En revanche, ce sens connoté avec la télicité constituent des propriétés essentielles du perfectif russe.

\subsection{Invariant du perfectif russe}

Les perfectifs qui marquent une évaluation de la durée (perfectif à préfixe délimitatif), de l'évolution du procès (perfectif par changement suffixal ou thématique de base) et l'inchoativité du procès (perfectif à préfixe inchoatif) ne comportent pas de télos. Par conséquent, la télicité ne peut pas être considérée comme une propriété fondamentale de l'aspect perfectif.

Quelle est donc la propriété commune à tous les emplois perfectifs (téliques et atéliques) ? Certains chercheurs considèrent qu'il n'y a pas d'invariant, puisqu'il s'agit d'une combinaison de paramètres de niveaux différents. Cela amène inévitablement à l'éclatement des valeurs aspectuelles spécifiques ( $\mathrm{c} f$., par exemple Timberlake, 1982 ; Mehlig, 1981 ; Zaliznjak \& Šmelëv, 1997). D'autres choisissent d'élargir la notion de télicité pour qu'elle puisse couvrir tous les emplois perfectifs en russe (Šeljakin, $2001: 215$, Bondarko, $1987: 50-51)^{6}$.

Je pense que la multitude d'emplois aspectuels contextuels et la complexité du problème n'annulent pas l'existence de l'invariant aspectuel et l'importance de sa définition. Cependant, la tendance à vouloir unifier à tout prix tous les emplois du perfectif sous l'étiquette du télos nuit à la compréhension et à la définition de la vraie nature de l'aspect russe en particulier et de l'aspect en général.

Je propose la définition suivante de l'invariant sémantique du perfectif russe : expression de l'évaluation de la durée délimitée du procès - de l'évaluation du résultat (télos) ou de l'évolution du déroulement (perception subjective de la durée) - ou expression de l'inchoativité du procès.

J'insiste ici sur évaluation du procès par le locuteur qui est, à mon avis, une propriété sémantique inhérente au perfectif délimitatif russe (télique et atélique). En effet, la constatation neutre de la durée du procès délimitée est marquée par l'imperfectif. Pour le perfectif russe, il convient de distinguer trois niveaux d'abstraction sémantique :

1) Durée délimitée (procès téliques et procès atéliques);

2) a) au début et à la fin ; b) au début sans marquer la fin ;

3) a) Évaluation du procès délimitée au début et à la fin (du résultat pour le procès télique, de la durée du procès atélique, de l'évolution du procès atélique constatée pat le locuteur au moment de l'énonciation) ; b) inchoativité/ingressivité du procès atélique délimité au début seulement.

Je conclus donc que la délimitation de la durée du procès reste la propriété fondamentale de tous les emplois du perfectif ${ }^{7}$. 


\section{La télicité et l'aspect délimité}

La durée délimitée est transmise en français par les temps grammaticaux (par exemple, passé composé, passé simple) qui marquent également l'aspect délimité. La question se pose de savoir si l'aspect délimité en français peut marquer également une évaluation de la durée, du résultat ou l'inchoativité/ingressivité du procès, comme c'est le cas du perfectif russe.

L'évaluation de la durée délimitée ne peut pas être exprimée par l'aspect délimité en français. En revanche, la télicité et l'inchoativité peuvent avoir une expression grammaticale.

Examinons tout d'abord les procès téliques. La télicité concerne deux types de procès : 1) procès à deux phases (durée + télos) : «lire un livre »;2) procès ponctuels (procès sans durée représenté par le seul télos) : « tressaillir ».

\subsection{Les procès duratifs téliques}

Les procès duratifs téliques sont à deux phases. La relation entre les phases peut concerner :

a) l'épuisement ou l'aboutissement de l'objet («j'ai mangé une pomme», «j'ai construit une maison ») ou du sujet («la neige a fondu ») (voir Verkuyl, 2000 ; Krifka, 1998 ; Filip, 1999 ; Dowty, 1991);

b) la transition quantité (durée) / qualité (télos).

La phase durative des deux types de procès est marquée en russe par l'imperfectif; la phase télique, par le perfectif du même verbe. L'expression des deux types de procès est différente en français : les deux phases du procès peuvent être exprimées par le même verbe ou par deux verbes différents.

\subsubsection{Les procès quantisés}

Les procès quantisés sont des procès évolutifs qui amènent à l'épuisement progressif de l'objet jusqu'à son anéantissement complet («manger une pomme») ou bien à la constitution progressive de l'objet jusqu'à son aboutissement complet («écrire une lettre»). L'épuisement/aboutissement de l'objet implique que le procès est télique. Cette interdépendance de l'«argument» et de la télicité est appelée composition aspectuelle. Elle a été initialement présentée dans la théorie méréologique de Krifka (1986, 1992, 1998) et dans la théorie aspectuelle de Verkuyl $(1999,2003)$. Ainsi, selon Krifka, l'argument et/ou le prédicat est cumulatif s'il est additif (en rajoutant du beurre au beurre, on obtient du beurre, des pommes aux pommes, on obtient des pommes ; marcher + marcher $=$ marcher). Si le prédicat n'est pas additif, il est quantifiable (si on rajoute une pomme à une pomme, on n'a plus une pomme, mais des pommes; pousser un cri + pousser un $c r i \neq$ pousser un cri). Autrement dit, le procès évolutif («dynamique » selon Filip, 1999) concerne l'évolution graduelle de la quantité de l'objet ou du sujet (« thème incrémental » de Dowty, 1991 ; «patient graduable» de Filip, 1999). Ainsi, Verkuyl considère que, si l'action porte sur un objet quantifiable (a house, two houses, the houses), le procès est télique et si l'action est dirigée vers un objet non quantifiable (houses, milk), le procès est atélique. Quant à Filip, elle formule la règle suivante de la télicité (1999: 94) :

$$
\begin{aligned}
& \text { verbe dynamique }+ \text { objet quantifiable graduable }= \\
& \text { groupe verbal télique (manger une pomme }- \text { T.M.) } \\
& \text { verbe dynamique }+ \text { objet cumulatif graduable }= \\
& \text { groupe verbal atélique (manger des pommes }- \text { T.M.). }
\end{aligned}
$$

Si l'on applique ce modèle au français, le rôle des déterminants devient primordial. ${ }^{8}$

Ainsi, selon la théorie de la composition aspectuelle, les procès quantisés sont constitués de deux phases : processus évolutif et télos (anéantissement/aboutissement de l'objet). L'objet est divisible et épuisable. Le 
télos du procès quantisé est atteint lorsque l'objet est épuisé. L'épuisement de l'objet est marqué par un déterminant.

Je remarquerai ici que la théorie de la composition aspectuelle considère la propriété télique/atélique comme une propriété aspectuelle fondamentale et donc suffisante à l'identification de l'aspect.

La télicité a-t-elle une expression formelle en français ? Pour répondre à cette question, appliquons le test de la télicité aux procès quantisés exprimés par les verbes français au passé. Ce test distributionnel consiste à utiliser les adverbes temporels "pendant quelque temps" (s'appliquant exclusivement aux procès atéliques) et « en quelque temps » (s'appliquant aux procès téliques) ${ }^{9}$. Il montre qu'en français, les deux phases - évolutive (9a) et télique (9b) - sont exprimées par la même forme verbale ${ }^{10}$ :

(a) J'ai écrit la lettre pendant cinq minutes. [on ne sait pas si la lettre est écrite ou non]

(b) J'ai écrit la lettre en cinq minutes. [la lettre est écrite]

Cependant, la phase télique du procès peut être rendue en français par des moyens grammaticaux mais de façon non exclusive : temps grammatical délimité (temps composé ou passé simple) et article défini du SN objet :

Hier soir, Paul a lu la lettre que je lui avais apportée. [la lettre est lue]

Si on changeait d'objet dans l'exemple (10) et qu'on ne lisait plus la lettre mais le livre, la situation changerait :

Hier soir avant de se coucher, Paul a lu le livre que je lui avais apporté. [on ne sait pas si le livre est lu ou non]

L'article indéfini transmettra le non-épuisement de l'objet de façon univoque ('le livre n'est pas lu') à condition que l'article indéfini n'ait pas la fonction d'un numéral :

Hier soir avant de se coucher, Paul a lu un livre.

Cependant, les situations (11) et (12) peuvent être interprétées comme téliques toutes les deux de façon univoque. Il suffit de changer l'intervalle temporel et de placer le procès dans un passé révolu :

(a) Paul a lu ce livre l'année dernière. [le livre est lu]

(b) L'année dernière, Paul a lu un livre. [le livre est lu]

En d'autres termes, l'interprétation télique du procès est conditionnée par plusieurs facteurs. Il me semble donc important de distinguer ici la télicité potentielle et la télicité réelle. La télicité potentielle (« télicité lexicale » de Comrie) concerne le plan conceptuel des procès. Ainsi « lire qqch » est potentiellement télique : tôt ou tard, ce quelque chose sera lu. Le terme de télicité lexicale de Comrie (2001 : 117) me semble mal approprié, puisqu'il devrait être utilisé sur le plan lexical et non sur le plan conceptuel. En effet, l'expression lexicale de la télicité veut dire que le radical d'un verbe exprime le sens télique (par exemple, arriver, résoudre sont toujours téliques, quel que soit leur contexte grammatical), ce qui n'est pas le cas du verbe lire. La télicité grammaticale (terme de Comrie) concerne la télicité dans un contexte linguistique approprié (par exemple, j'ai déjà lu un livre). Cependant, le seul contexte grammatical est souvent insuffisant, il est indispensable de tenir compte de la situation. La télicité réelle est la télicité du verbe dans un contexte non seulement linguistique mais aussi situationnel. La situation réelle, notre connaissance du monde jouent un rôle important (par exemple, on lit une lettre en une fois, mais le livre en plusieurs séquences ; on connaît une durée approximative de la lecture d'un livre, etc.).

Autrement dit, les termes télicité lexicale et télicité grammaticale sont réservés, à mon avis, à l'expression formelle de la télicité, tandis que la télicité potentielle et la télicité réelle appartiennent au niveau conceptuel.

Je pense donc que la théorie de la composition aspectuelle ne peut pas être appliquée de façon mécanique au français. En effet, elle fait confusion, à mon avis, entre télicité potentielle et télicité réelle. L'interprétation télique du procès n'est pas conditionnée uniquement par l'épuisement de l'objet défini, mais aussi et surtout par notre connaissance du monde. Autrement dit, l'interprétation télique de (10a) ('la 
lettre est lue') est due non seulement à la définitude du SN objet (on sait de quelle lettre il s'agit), mais aussi à la situation (on lit une lettre en une seule fois).

Il convient ici d'aborder le problème du statut particulier du passé simple par rapport aux procès quantisés. En effet, selon Vetters (1996:131), «personne ne met en question la perfectivité du passé simple ». La «perfectivité » présuppose l'actualisation de la télicité des procès. En effet, le passé simple déclenche prioritairement une lecture télique (14), sauf indication contraire (15) :

La veille de son départ, avant de se coucher, elle lut le livre qu'il lui avait apporté. (l'interprétation télique 'le livre est lu' est prioritaire, mais l'interprétation atélique 'le livre n'est pas lu' n'est pas exclue)

La veille de son départ, avant de se coucher, elle lut pendant quelque temps le livre qu'il lui avait apporté. [le livre n'est pas lu]

J'en déduis que la télicité des procès du passé simple est plutôt un effet de sens que la vraie propriété de ce temps grammatical. En effet, dans l'exemple (14), il s'agit d'un fait vu a posteriori dans un registre narratif. J'emprunte ce terme à G. Guillaume pour désigner un sens produit par un ensemble de facteurs situationnels par opposition au sens inhérent à un temps grammatical donné. Il n'en reste pas moins que cet effet de sens télique est à l'origine d'une propriété aspectuelle, spécifique du passé simple, de marquer la conséquence des procès délimités. En effet, la télicité et la succession des procès sont interdépendants. Rappelons que Jakobson, le fondateur de la théorie de l'ordre des procès (taxis), souligne que la conséquence des procès est exprimée en russe par une suite de perfectifs, et la coïncidence par la suite des imperfectifs (Jakobson, 1965). La théorie de Jakobson est applicable au français. En effet, l'une des propriétés essentielles du perfectif russe est la télicité. La télicité et l'atélicité peuvent avoir une expression lexicale en français, par exemple résoudre (télique), marcher (atélique). Ainsi l'infinitif français, tout comme l'infinitif russe, peut être porteur dans certains cas du sens télique ou du sens atélique, indépendamment de son expression temporelle : se lever et partir (succession des procès téliques), regarder et sourire (coïncidence des procès atéliques) ${ }^{11}$.

Ainsi, l'ordre des procès (taxis) est étroitement lié à l'aspect (perfectif/imperfectif) en russe et à la télicité/atélicité en français. De plus, en français, les temps composés expriment de façon systématique la relation d'antériorité. Autement dit, l'ordre des procès en général est exprimé, d'une part, par l'aspect et, d'autre part, par les temps grammaticaux d'antériorité. Maslov, aspectologue russe travaillant sur la typologie de l'aspect, remarque à ce sujet $(1984: 26)$ :

\begin{abstract}
Dans plusieurs langues le taxis est couplé soit avec le temps, soit avec l'aspect pour former une seule catégorie grammaticale. L'association du taxis et du temps donne une structure temporelle complexe (à deux, et parfois à plusieurs niveaux) exprimée dans certaines langues par des formes spéciales faisant partie de l'ensemble des temps dits relatifs. ${ }^{12}$
\end{abstract}

Autrement dit, le russe compense l'absence des temps composés par l'aspect qui exprime l'ordre des procès. En français, la situation est plus complexe : l'ordre des procès est transmis régulièrement par les temps grammaticaux, mais l'opposition délimité/non délimité est une opposition aspectuelle ${ }^{13}$ qui peut engendrer la télicité comme effet de sens :

(16) Dès qu'il a eu déjeuné, il s'en est allé. (Exemple de Guillaume 1929) [le déjeuner est mangé]

En français, l'antériorité est donc couplée avec le temps, mais l'évaluation de la durée du procès ou l'interprétation télique sont des propriétés aspectuelles résultant de l'opposition délimité/non délimité. Ainsi, la spécificité du passé simple est d'exprimer de façon univoque non seulement la succession, mais en particulier la postériorité des procès successifs. Cette propriété du passé simple est signalée par Vetters (1996 : 141) qui donne des exemples suivants :

(a) Jane me quitta. Elle tomba amoureuse de quelqu'un d'autre. (Postériorité)

(b) Jane m'a quitté. Elle est tombée amoureuse de quelqu'un d'autre. (Postériorité ou antériorité)

Il est à noter qu'en russe, une suite de procès passés au perfectif marque la conséquence inférée de l'ordre chronologique de leur déroulement ${ }^{14}$. Je pense que cette propriété de l'aspect perfectif se retrouve dans le 
sens aspectuel du passé simple. En effet, dans l'exemple (17a), le départ de Jane est à l'origine du fait qu'elle tombe amoureuse par la suite. Par conséquent, cette propriété du passé simple de marquer la postériorité des procès est une valeur aspectuelle (et non temporelle); elle provient du registre narratif du passé simple qui présente les faits a postériori dans un ordre chronologique.

\subsubsection{La transition quantité/qualité}

Les procès dits cumulatifs (par exemple se promener, se reposer) sont des procès évolutifs additifs: « marcher » + "marcher » = « marcher » (Krifka, 1992). Les recherches sur la télicité dans les langues analytiques, de préférence en anglais, démontrent que le procès cumulatif n'est jamais télique (Krifka, 1992 ; Filip, 1999 ; Verkuyl, 1999). Cependant, en russe, la situation est différente : les procès cumulatifs peuvent abouir à un télos, c'est-à dire que la phase quantitative de la durée peut être suivie par la phase qualitative du résultat. Cette transition quantité/qualité dans le même procès se présente dans les langues slaves par deux formes aspectives du même verbe: quantité (imperfectif)/ qualité (perfectif). Par exemple : rešat'/rešit' (zadaču) " chercher/trouver la solution (d'un problème)», ubeždat'/ubedit' « essayer de convaincre / parvenir à convaincre ». Cette transition est typique de l'aspect russe.

En français, la phase qualitative est conceptualisée comme un procès autonome télique : convaincre, résoudre. Ces verbes ne peuvent pas avoir une interprétation atélique durative :

(18) *Il a résolu ce problème pendant dix minutes.

Cependant, ces procès comportent une durée qui précède le télos :

(19) Il a résolu ce problème en dix minutes.

Sur le plan conceptuel, les procès de transition sont différents des procès quantisés (voir § 3.1.1.). Par exemple, Pierre a mangé la pomme en dix minutes veut dire que le procès de «manger une pomme » a duré dix minutes et qu'au bout des dix minutes, au moment précis de l'anéantissement de la partie comestible de la pomme, le télos du procès a été atteint. Par contre, la relation entre les deux phases des procès de transition quantité/qualité est moins mécanique. Ainsi, Pierre m'a convaincu en cinq minutes ne veut pas dire que Pierre a essayé de me convaincre pendant cinq minutes (il est possible qu'il ait mis beaucoup plus de temps pour apporter ses arguments), mais que ces cinq dernières minutes se sont révélées décisives pour que je sois enfin convaincu. Autrement dit, les deux formes aspectives russes se trouvant en relation quantité/qualité soulignent tout simplement qu'une partie ou la totalité de la phase quantité (durée) est indispensable à la réalisation de la phase qualité (télos), les deux phases étant perçues comme faisant partie d'un même procès. La relation processus/résultat entre les procès n'est pas toujours conceptualisée comme étant la transition quantité/qualité entre les deux phases du même procès. Par exemple, l'opposition lexicale « chercher » / «trouver» (processus/résultat) est perçue en russe comme deux procès distincts : iskat'/najtit ${ }^{15}$.

Cependant, il convient d'apporter quelques précisions. Il existe une différence fondamentale entre ces deux types d'expression de l'aspect: grammaticale en russe (imperfectif/perfectif du même verbe) et lexicale en français (processus/résultat transmis par deux lexèmes). En effet, le perfectif télique russe ne peut pas avoir une interprétation durative, ce qui implique que le perfectif est incompatible avec le présent. Pour marquer la valeur télique itérative au présent, on recourt à l'imperfectif. En revanche, les verbes français (résoudre, obtenir, condamner, etc.) expriment la télicité lexicalement (par leur radical), ce qui implique que la télicité unique aux temps délimités comme le passé composé (20a) ou la télicité répétitive aux temps non délimités comme le présent et l'imparfait (20b) sont exprimées toutes les deux par le même verbe ${ }^{16}$ :

(20) (a) J'ai résolu ce problème.

(b) Je résous toujours ce type de problèmes. 


\subsection{Les procès ponctuels}

Les procès ponctuels sont des procès conceptualisés comme infiniment courts, qui n'ont pas de durée (par exemple, tressaillir). La conceptualisation d'un procès en tant que ponctuel ou duratif n'est pas toujours en accord avec sa durée réelle dans le temps. Bache (1982) remarque à ce sujet que la plupart des verbes ponctuels ne désignent pas des procès instantanés et que ces procès ont une certaine durée, même si elle est très courte.

Le caractère ponctuel d'un procès est exprimé en français lexicalement, c'est-à-dire par le radical du verbe. Il y a deux types de procès ponctuels :

1) uniques: ceux qui, de par leur nature, ne sont pas susceptibles de se répéter, puisqu'ils sont imprévisibles, survenus indépendamment de la volonté du sujet (tressaillir, expirer 'mourir') ;

2) semelfactifs : ceux qui peuvent se multiplier et se transformer en procès multiplicatifs et désigner une suite discontinue de phases équivalentes d'un même procès (frissonner, étinceler).

Les procès ponctuels uniques sont téliques. En français leur télicité est exprimée lexicalement, par le radical du verbe (en russe, la télicité lexicale des verbes est doublée par la télicité grammaticale, par l'aspect perfectif). La télicité de ce type des verbes en russe et en français conditionne leurs propriétés communes :

(i) ils sont incompatibles avec le présent actuel qui marque le processus se déroulant au moment de son observation (Apresjan 2003 : XIX) (21) :

(21) *Je vois qu'il expire.

(ii) ils ne peuvent pas avoir de complément de durée (22) :

(22) * Il expira pendant une heure.

Il est possible parfois d'utiliser un complément de durée ponctuelle en une seconde ou bien en quelques secondes où le nombre indéfini de secondes ne représente pas une durée réelle, mais correspond à un moment ponctuel.

(iii) ils peuvent marquer le passé récent (23) :

(23) Vous vous trompez. ('vous venez de vous tromper').

En revanche, les procès semelfactifs/multiplicatifs ont une expression différente en russe et en français. En russe, cette relation est marquée grammaticalement : «crier» kričat' (imperfectif multiplicatif) / «pousser un cri » kriknut' (perfectif semelfactif).

En français, les procès semelfactifs et les procès multiplicatifs sont exprimés par les mêmes formes verbales. L'interprétation semelfactive (24a) ou multiplicative (24b) s'opère en fonction du contexte grammatical et situationnel :

(a) Elle entendit un bruit et frissonna.

(b) Elle frissonna pendant quelques secondes.

Il est toutefois à noter que l'aspect délimité déclenche prioritairement la lecture semelfactive (25a) sauf indication contraire (24b). En revanche, l'aspect non délimité déclenche une interprétation multiplicative (25b) :
(a) Elle a frisonné.
(b) Elle frisonnait.

Il est possible aussi en français d'exprimer de façon univoque la valeur semelfactive : avoir un frisson, faire un saut, pousser un $\mathrm{cri}^{17}$. 


\subsection{Les procès inchatifs/ingressifs}

Rappelons que l'inchoativité et l'ingressivité sont atéliques. Les procès inchoatifs marquent le commencement du procès de façon neutre; tandis que les procès ingressifs sont connotés: le commencement du procès intense est perçu comme brutal et inattandu (voir § 2.3.).

L'inchoativité du procès est exprimée en français par un temps délimité et par un circonstant :

$$
\text { Dès qu'il m'a vu, il m'a haï. }
$$

Ainsi, l'interprétation inchoative du procès « haïr » de l'exemple (26) est due au circonstant dès qu'il m'a $v u$.

Le sens ingressif peut être transmis en français par le passé simple s'il s'agit des verbes d'état (Guenthner, Hoepeloman \& Rohrer, 1978 ; Vetters, 1996 : 109) :

$$
\text { Après le troisième verre, il fut ivre mort. (Exemple de Vetters 1996) }
$$

\subsection{Bilan}

L'interprétation télique du procès exprimé par un temps délimité en français est le résultat de l'ensemble des paramètres : aspect délimité du verbe, définitude du SN objet et situation extralinguistique (nature de l'objet ou du sujet, relativité de l'intervalle temporel).

Le passé simple a une valeur aspectuelle, il marque :

1) la postériorité des procès

2) l'ingressivité des états.

Cependant, la télicité des procès n'est pas systématique, elle est un effet de sens.

\section{L'ordre des procès et l'aspect non délimité}

L'aspect non délimité pour le tiroir du passé est marqué en français par l'imparfait. Il s'agit dans ce cas de la non-délimitation relative. En effet, le procès duratif a un début et une fin, mais il est non délimité par rapport à un autre intervalle temporel délimité focalisé dans l'énonciation. Par exemple :

(26) Quand je suis rentré, elle était au téléphone.

Dans l'exemple (26), les limites temporelles du procès englobant (aspect non délimité) ne sont pas importantes pour le locuteur, puisque la focalisation porte sur le procès englobé (aspect délimité).

On remarquera que la non-délimitation absolue (lorsque le procès n'a pas de début, ni de fin) n'est pas transmise par l'aspect, mais par des moyens lexicaux (verbes d'état et des circonstants, par exemple, toujours) :

(27) J'ai toujours aimé la promenade dans la forêt

La relativité de l'aspect non délimité permet de marquer la relation de coïncidence des procès :

(28) Nous étions tous au jardin quand retentirent les deux coups hésitants de la clochette (Poust, Du coté de chez Swann)

(29) Fernande, qui habitait dans l'arrière boutique, était en train de se préparer à dîner. (Druon, Les grandes familles)

Cette thèse aspectuelle semble être compromise en français par l'emploi de l'imparfait de narration qui relève du registre narratif (voir, par exemple Molendijk, 1990 ; Berthonneau, 2000) :

$$
\text { À huit heures précises, le général expirait. (Exemple de Leeman 1996) }
$$


En effet, il s'agit ici d'un procès télique, et donc délimité. Cependant, l'imparfait a ici une valeur spécifique, justement marquée grâce à cette contradiction entre l'aspect non délimité et la durée délimitée du procès : l'imparfait accentue l'importance et la solennité du procès ponctuel (30). L'aspect non délimité marque donc en français une évaluation subjective de l'importance du procès. Rappelons que l'opposition de l'évaluation subjective du procès par rapport à la constatation neutre est marquée en russe par une opposition aspectuelle.

L'imparfait dans les emplois standard exprime donc le procès non délimité. En revanche, l'imparfait de narration peut dénoter le procès délimité, ce qui implique qu'il peut marquer également la relation de postériorité immédiate et de postériorité décalée.

L'imparfait de postériorité immédiate accentue la relation d'inférence entre les procès (je souligneT.M.) :

Jean tourna l'interrupteur. La lumière l'éblouissait. (Exemple de Berthonneau 2000)

(32) Elle revint au bout d'un moment me dire qu' [...] on trouverait le moyen de la [ma lettre] faire passer à maman. Aussitôt mon anxiété tomba [...]. Maintenant, je n'étais plus séparé d'elle; les barrières étaient tombées, un fil délicieux nous réunissait. (Proust, Du coté de chez Swann.)

L'imparfait de postériorité décalée (imparfait de rupture) déclenche une connotation qui résulte du décalage perçu comme trop court entre les procès et de l'accélération du passage d'un procès vers l'autre (je souligne - T.M.) :

Il était prêt, en effet, quand le vieux serviteur reparut, et comme il avait en poche de ces arguments qui donnent des jambes aux pires chevaux de fiacre, moins de dix minutes plus tard, il arrivait à l'Hôtel des Folies. (Gaboriau, L'argent des autres - La pêche en eau trouble)

Comme elle avait été à l'Opéra, une nuit d'hiver, elle rentra toute frissonnante de froid. Le lendemain, elle toussait. Huit jours plus tard, elle mourait d'une fluxion de poitrine. (Maupassant, Contes et nouvelles, exemple de Berthonneau 2000)

Berthonneau (2000) souligne que l'imparfait de rupture produit un effet de dynamisme et d'accélération, qu'il pourrait être remplacé par le passé simple et se caractérise par une «chronologie serrée (le lendemain, le soir même, un an après, etc.) », que l'événement à l'imparfait est perçu comme le dernier de la série, ce qui est indispensable à l'interprétation de rupture. Les procès sont en relation de causalité inférée, le dernier procès de la série est conclusif, il clôt la situation.

Je pense que ces caractéristiques de l'imparfait de narration renforcent la thèse aspectuelle plutôt qu'elles ne l'affaiblissent. En effet, l'ordonnancement de procès dans le discours se réalise non seulement en fonction de la relation temporelle objective, mais aussi en fonction de la relation logique établie par le locuteur ainsi que de l'évaluation subjective qu'il fait (voir Jakobson, 1965).

Par conséquent, l'aspect non délimité s'applique non seulement aux procès à durée non délimitée, mais aussi aux procès à durée délimitée. Lorsque les procès à durée délimitée sont marqués par l'aspect non délimité, ils acquièrent des valeurs aspectuelles spécifiques.

Ainsi, l'imparfait de narration présente deux valeurs aspectuelles fondamentales :

1) évaluation subjective de l'importance du procès (30);

2) expression de l'ordre spécifique des procès: de postériorité décalée $(33,34)$ ou de postériorité immédiate inférée $(31,32)$.

L'aspect non délimité français a donc deux sens :

1) sens non délimitatif relatif non connoté (dans les emplois standard);

2) sens délimitatif connoté (dans les emplois spécifiques de narration, conditionnés par l'ordre des procès). 


\section{Conclusion}

L'opposition systématique de l'expression verbale de la durée délimitée par rapport à la durée relativement non délimitée du procès peut être considérée comme la propriété fondamentale de la catégorie de l'aspect exprimé au passé par l'opposition des temps composés ou le passé simple par rapport à l'imparfait. Par conséquent, l'aspect est une catégorie flexionnelle du verbe français.

L'opposition aspectuelle permet d'exprimer non seulement la relation temporelle objective entre les procès, mais aussi la perception de la durée et son évaluation subjective, ainsi que d'accentuer les relations ontologique et logique de l'ordre des procès pour exprimer leur perception.

Le jeu de deux aspects s'appliquant au procès de nature différente (télique/atélique, délimité/non délimité) permet de différencier les emplois neutres et les emplois connotés. Cependant, il faut remarquer que la valeur connotative de l'opposition aspectuelle s'applique de préférence au français de narration.

\section{Références bibliographiques}

Apresjan, Ju.D. (2003). Lingvističeskaja terminologija slovarja. In Ju.D. Apresjan, ed. Novyj ob"jasnitel'nyj slovar' sinonimov russkogo jazyka. Tretij vypusk. Moscou : Jazyki slavjanskoj kul’tury, XVIII-LI.

Arrivé, M. \& al. (1986). La grammaire d'aujourd'hui: guide alphabétique de linguistique française. Paris: Flammarion.

Bache, C. (1982). Aspect and Aktionsart: towards a semantic distinction. Journal of linguistics, 18, 57-72.

Berthonneau, A.-M. (2000). L'imparfait de narration dans tous ses états. L'Imparfait: philologie électronique et assistance à l'interprétation des textes. Reims : Presses Universitaires de Reims, 73-109.

Bondarko, A.V. (1987). Soderžanie i tipy semantiki predela. In A.V. Bondarko \& al., eds. Teorija funkcional'noj grammatiki, $3^{\mathrm{e}}$ éd. Moscou : URSS, 2003, 46-51.

Bosveld-de Smet, L. (2000). Les syntagmes nominaux en des et $d u$ : un couple curieux parmi les indéfinis. In L. Bosveld-de Smet, M. Van Petegnem, D. Van de Velde, éds. De l'indétermination à la quantification: les indéfinis. Artois : Artois Presses Université, 16-116.

Boukreeva, T. (1996). L'alternance et la supplétion lexicales (étude comparative du français et du russe). In Fougeron Irina, éd. Études russes. Villeneuve d'Ascq : Presses Universitaires du Septentrion, 137-149.

Breu, W. (2004). Teoretičeskaja model' vzaimodejstvija vida s leksikoj. In M.L. Remnëva \& A.A. Polikarpov, eds. Russkij jazyk: istoričeskie sud'by i sovremennost' : II Congrès International des russistes. Moscou : Presses Universitaires de Moscou, 251-252.

Bulygina, T.V. (1982). K postroeniju tipologii predikatov v russkom jazyke. In Semantičeskie tipy predikatov. Moscou : Nauka, 7-85.

Cohen, D. (1989). L'aspect verbal. Paris : PUF.

Comrie, B. (1976). Aspect. An introduction to the study of verbal aspect and related problems. Cambridge: Cambridge University Press.

Comrie, B. (1989). Perfectif et télique, Travaux de linguistique, 19, 57-66.

Comrie, B. (2001). Obščaja teorija glagol'nogo vida. In M.Ju. Čertkova, ed. Trudy aspektologičeskogo seminara filologičeskogo fakul'teta MGU im. M.V. Lomonosova, 1. Moscou : Presses Universitaires de Moscou, 115-121.

Čertkova, M.Ju. (2004). Tipologija i èvoljucija funkcional'no-strukturnyh modelej kategorii vida/aspekta (na materiale sovremennyh russkogo, francuzskogo, švedskogo, finskogo i japonskogo jazykov). In M.Ju. Čertkova, ed. Trudy aspektologičeskogo seminara filologičeskogo fakul'teta MGU im. M.V. Lomonosova, IV. Moscou: Presses Universitaires de Moscou, 183-252.

Dowty, D. R. (1991). Thematic proto-roles and argument selection. Langage, 67/3, 547-619.

Dubois, J. \& al. (1994). Dictionnaire de linguistique et des sciences du langage. Paris : Larousse.

Filip, H. (1999). Aspect, eventuality types and noun phrase semantics. New York, London : Garland Publishing. 
Holt, J. (1943). Études d'aspect. Acta Jutlandica, 15/2, 1-84.

Gosselin, L. (1996). Sémantique de la temporalité en français : un modèle calculatoire et cognitif du temps et de l'aspect. Louvain-la-neuve : Duculot.

Gosselin, L. (2005). Temporalité et modalité. Bruxelles : Duculot.

Guenthner, F., Hoepelman, J. \& Rohrer, Ch. (1978). A note on the passé simple. In Ch. Rohrer, ed. Paper on Tense. Aspect and Verb Classification. Tübunbgen: Narr Verlag.

Guillaume, G. (1929). Temps et verbe: théorie des aspects, des modes et des temps. Paris : Librairie Honoré Champion, 1970.

Guillaume, G. (1951). La représentation du temps dans la langue française. Langage et science du langage. Paris / Québec : Librairie A. Nizet / Presses de l'Université Laval, 1964, 184-207.

Imbs, P. (1965). L'emploi des temps verbaux en français moderne. $4^{\mathrm{e}}$ ed. Paris : Klincksieck.

Jacobs, R. A. \& Rosenbaum, P. S. (1968). English transformational grammar. Waltham, Massachussets: Blaisdell Publishing Co.

Jakobson, R. (1965). A la recherche de l'essence du langage. Diogène, 51, 22-38.

Klum, A. (1961). Verbe et adverbe. Stockholm / Göteborg / Uppsala : Almqvist \& Wiksell.

Krifka, M. (1986). Nominalreferenz und Zeitkonstitution. Zür Semantik von Massentermen, Individualtermen, Aspektklassen, PhD Dissertation. München : Universität München.

Krifka, M. (1992). Thematic relations as links between nominal reference and temporal construction. In I. A. Sag, A. Szabolsci, eds., Lexical matters, CSLI Lecture Notes. Stanford : CSLI Publications, 29-53.

Krifka, M. (1998). The origins of telicity. In S. Rothstein, ed. Events and grammar. Dorbrecht - Boston - London: Kluwer Academic Publishers, 197-235.

Kupfermann, L. (1979). L'article partitif existe-t-il ? Le Français Moderne, 41/1, 1-16.

Laurendeau, P. (1995). Exploitations du cadre de la théorie des repérages énonciatifs en linguistique descriptive : le cas du tiroir de l'imparfait. In J. Bouscaren, J.-J. Franckel, S. Robert (dir.), éds. Langues et langage. Problèmes et raisonnement en linguistique - Mélanges offerts à Antoine Culioli. Paris : Presses Universitaires de France, 331343.

Leeman, D. (1996). Grammaire du verbe français. Paris : Nathan.

Lerat, P. (1981). L'aspect dans le lexique français contemporain. Cahiers de lexicologie, 39, 48-54.

Lyons, J. (1970). Linguistique générale : introduction à la linguistique théorique, trad. fr. Paris : Larousse.

Maslov, Ju.S. (1978). Stat'i po aspektologii. In Ju.S. Maslov. Izbrannye trudy : Aspektologija. Obščee jazykoznanie. Moscou : Jazyki slavjanskoj kul'tury, 2004, 303-517.

Maslov, Ju.S. (1984). Očerki po aspektologii. In Ju.S. Maslov. Izbrannye trudy : Aspektologija. Obščee jazykoznanie. Moscou : Jazyki slavjanskoj kul'tury, 2004, 18-302.

Mehlig, H. R. (1981). Satzsemantik und Aspektsemantik im Russischen (Zur Verbklassifikation von Zeno Vendler). Slavistischen Beiträgen, 147. München : Verlag Otto Sagner, 95-151.

Milliaressi, T. (2006). L'information aspectuelle dans un dictionnaire explicatif bilingue (sur l'exemple du russe et du français). In E. Corino, C. Marillo, C. Onesti, eds. Atti del XII Congresso Internazionale di Lessicografia, Torino, Proceedings XII EURALEX International Congress, I. Alessandria : Edizioni dell'Orso, 549-560.

Milliaressi, T. (2009). Temps / aspect : la traduction de la postériorité immédiate des procès passés. In T. Milliaressi, éd. La traduction : philosophie, linguistique et didactique, coll. UL3 Travaux et recherches. Villeneuve d'Ascq: Éditions du Conseil Scientifique de l'Université Charles-de-Gaulles - Lille 3, 135-138.

Milliaressi, T. (2010). Eščë raz o vidovom i o leksičeskom značenii pristavki po-. In M. Remneva, A. Polikarpov, eds. Russkij jazyk: istoričeskie sud'by i sovremennost': IV Congrès International des russistes. Moscou: Presses Universitaires de Moscou, 824-825. 
Molendijk, A. (1996). Anaphore et imparfait: la référence globale à des situations présupposées ou impliquées. Cahiers Chronos, 1, 109-123.

Peškovskij, A.M. (1956). Russkij sintaksis v naučnom osveščenii. $7^{\mathrm{e} e ́ d . ~ M o s c o u ~: ~ U c ̌ p e d g i z . ~}$

Smith, C. S. (1986). A speaker-based approach to aspect. Linguistics and Philosophy, 9, 97-115.

Šeljakin, M.A. (2001). O spornyh voprosah russkoj aspektologii. In M.Ju. Čertkova, ed. Trudy aspektologičeskogo seminara filologičeskogo fakul'teta MGU im. M.V. Lomonosova, I. Moscou: Presses Universitaires de Moscou, 210-219.

Timberlake, A. (1982). Invariance and the syntax of Russian aspect. In P.J. Hopper, eds. Tense-aspect: between semantics \& pragmatics. Amsterdam - Philadelphia: John Benjamins Publishing Co., 305-331.

Van de Velde, D. (1995). Le spectre nominal, des noms de matières aux noms d'abstraction. Louvain : Peeters.

Verkuyl, H. J. (1999). Aspectual issues. Structuring time and quantity 98. CSLI Lecture Notes, Stanford, California: CSLI Publications.

Verkuyl, H. J. (2000). Events as dividuals: aspectual composition and event semantics. In J. Higginbotham, F. Pianesi, A.C. Varzi, eds. Speaking of Events. New York/Oxford: Oxford University Press.

Verkuyl, H. J. (2003). Aspectual asymmetry and quantification. In J. Gutiérrez-Rexach, ed. Semantics. Critical concepts in linguistics 4. London/New York: Routledge, 71-103.

Vet, C. (1980). Temps, aspects et adverbes de temps en français contemporain. Genève : Librairie Droz.

Vetters, C. (1996). Temps, aspect et narration. Amsterdam - Atlanta : Editions Rodopi B.V.

Zaliznjak, A.A. \& Šmelëv, A.D. (1997). Lekcii po russkoj aspektologii. Slavistiche Beiträge 353, Bd 7. München : Verlag Otto Sagner.

${ }^{1}$ Les préfixes à sens lexical sont caractéristiques du russe. Par exemple, la direction du mouvement, exprimée en français par un lexème (arriver/partir) est traduite en russe par un préfixe (prihodit'/uhhodit') (le radical -hod- signifie le déplacement à pied).

${ }^{2}$ Ici et plus loin les caractères cyrilliques sont translittérés en caractères latins selon la norme internationale des slavistes qui reprend la recommandation ISO/R9 (version 1968) de l'Organisation internationale de normalisation, à l'exception de la lettre $x$ translittérée $h$.

${ }^{3}$ Il s'agit ici d'une supplétion grammaticale des bases lorsque les formes grammaticales du même mot se caractérisent par l'identité sémantique résultant de leur évolution diachronique (Boukreeva 1996).

${ }^{4}$ Je tiens à souligner que le perfectif russe d'un verbe duratif ne se résume pas à un télos sans durée, par opposition aux verbes ponctuels (voir 3.2). En effet, les verbes téliques duratifs peuvent avoir des circonstants de durée (Ja pročital knigu za dva časa «j'ai lu ce livre en deux heures ».) à l'inverse des verbes ponctuels (*On skončalsja $z a d v a$ časa « il est décédé en deux heures ».).

${ }^{5}$ Sur la propriété contrôlable/non contrôlable, voir (Bulygina 1982).

${ }^{6}$ Ainsi Bondarko modifie la notion même de télos pour concilier les différences sémantiques que présente l'aspect perfectif: à côté du télos au sens traditionnel qu'il nomme télos absolu, il introduit une notion de télos relatif (Bondarko 1987 : 50-51). Par exemple, le télos relatif est atteint dans le procès « les prix ont baissé », puisque les prix vont continuer à baisser après avoir atteint une limite relative.

${ }^{7}$ Pour la concision du propos, je n'analyse pas ici les emplois spécifiques de l'imperfectif télique russe.

${ }^{8}$ Sur les déterminants des et $d u$ en français, voir (Bosveld-de Smet 2000), (Van de Velde 1995).

${ }^{9}$ Selon Kupferman (1979) et Vet (1980), ce test est valable pour le français.

${ }^{10}$ Le russe recourt à deux formes aspectives différentes : Ja pisal (imperfectif) pis'mo pjat' minut « j'ai écrit la lettre pendant cinq minutes » / Ja napisal (perfectif) pis'mo za pjat' minut «j'ai écrit la lettre en cinq minutes ».

${ }^{11}$ En français la télicité est exprimée lexicalement, en russe grammaticalement (imperfectif/perfectif) et lexicalement (lorsque le radical du verbe est porteur de sens télique). 
12 Je traduis - T.M.

${ }^{13}$ Comparez avec (Guillaume $\left.1951: 189\right)$ : «La distinction de la forme simple et de la forme composé [...] est une distinction non pas de temps mais d'aspect et c'est à tort que les grammairiens, pour la plupart, la font entrer, ce qui embrouille les choses, dans le système de temps. »

${ }^{14}$ Sur le taxis en français et en russe, voir (Milliaressi 2009).

${ }^{15} \mathrm{La}$ relativité de la conceptualisation d'un même procès en tant que procès unique ou en tant que procès à deux phases peut être illustrée par deux verbes synonymiques : umirat'lumeret' (où l'imperfectif dénote la phase finale de la vie « être en train de mourir », tandis que le perfectif veut dire «être mort ») et le perfectivum tantum skončat'sja « décéder » (où le moment de la mort est conceptualisé comme un événement autonome irrévocable et unique ne faisant pas partie de la vie qui est un autre procès).

${ }^{16}$ En russe le procès télique unique est exprimé par le perfectif et le procès télique répétitif, par l'imperfectif.

${ }^{17}$ En russe, la valeur semelfactive est exprimée par le suffixe - $n u$ - qui perfectivise l'imperfectif multiplicatif : drožat' (imperfectif) / drognut ' (perfectif) « frissonner », stučat' (imperfectif) / stuknut ' (perfectif) «frapper (à la porte) », etc. 